\title{
STABILITY OF EQUILIBRIUM SOLUTIONS FOR THE FISHER EQUATION*
}

\author{
BY \\ JAMES M. GREENBERG \\ State University of New York at Buffalo and National Science Foundation
}

1. Introduction. In recent years there has developed an extensive literature on the Fisher equation:

$$
\partial u / \partial t=\partial^{2} u / \partial x^{2}+f(u) .
$$

An extensive bibliography may be found in Fife [1]. This equation, with appropriately chosen $f$ 's, may be used to describe populations of diploid individuals as well as certain flame propagation phenoma. Much of this work concerns the temporal stability of traveling wave solutions to (DE); for details see Fife and McLeod [2], Aronson and Weinberger [3], and Bramson [4].

Our concern here is somewhat different. We are interested in the stability, or lack thereof, of certain nonconstant equilibrium solutions to (DE). We shall confine our attention to what the geneticists refer to as the heterozygote inferior case, namely the situation when $f$ is given by

$$
f(u)=u(u-a)(1-u) \text { and } 0<a<\frac{1}{2} .
$$

These equilibrium solutions satisfy the time-independent version of (DE), namely the equation

$$
\frac{d^{2} u}{d x^{2}}+u(u-a)(1-u)=0, \quad x>0 \quad \text { and } \quad 0<a<\frac{1}{2}
$$

and the boundary conditions

$$
\frac{d u}{d x}(0)=0 \quad \text { and } \quad \lim _{x \rightarrow \infty} u(x)=0
$$

and are given by

$$
u=u_{\mathrm{eq}}(x)=\frac{6 a}{(1+a)\left(1-\frac{9 a}{2(1+a)^{2}}\right)^{1 / 2}} \frac{e^{a^{1 / 2} x}}{\left(1+\frac{2 e^{a^{1 / 2} x}}{\left(1-\left[9 a / 2(1+a)^{2}\right]\right)^{1 / 2}}+e^{2 a^{1 / 2} x}\right)}
$$

* Received October 9, 1980. This work was partially supported by the National Science Foundation under Grant \# MCS80-18531. 
Our basic result is that the variational operator

$$
(L \phi)(x) \stackrel{\text { def }}{=} \frac{d^{2} \phi}{d x^{2}}(x)-\left(3 u_{\mathrm{eq}}^{2}(x)-2(1+a) u_{\mathrm{eq}}(x)+a\right) \phi(x)
$$

acting on functions satisfying

$$
\frac{d \phi}{d x}(0)=0 \quad \text { and } \quad \lim _{x \rightarrow \infty} \phi(x)=0
$$

has exactly one positive eigenvalue, call it $\mu a$, and an associated eigenfunction which is positive. This result is not new. What is new, and our primary reason for writing this note, are the estimates for the eigenvalue and the methodology we use to obtain these estimates. A sequence of algebraic equations for the approximate eigenvalue is obtained and these equations yield a sequence of approximate eigenvalues $\left\{\mu_{n} a\right\}_{n=1}^{\infty}$ which converge monotonically from below to the desired quantity. Such results are rare for infinite interval problems.

Our program for the remainder of this paper is as follows. In Sec. 2 we establish some qualitative results for the variational operator. These results establish the existence of a unique positive eigenvalue for the variational operator. In Sec. 3 we develop and analyze the tools to calculate this quantity. These are the new results.

2. Qualitative results. We start with some observations about the nonconstant equilibrium solution to (DE) and (BC). It satisfies

$$
\begin{gathered}
d^{2} u / d x^{2}+u(u-a)(1-u)=0, \quad x>0, \\
\frac{d u}{d x}(0)=0 \quad \text { and } \quad \lim _{x \rightarrow \infty} u(x)=0 .
\end{gathered}
$$

The phase portrait for $(\mathrm{DE})_{\text {eq }}$ is shown in Fig. 1. The orbit associated with the equilibrium solution is the curve connecting $\left(u_{-}, 0\right)$ to $(0,0)$ lying in the halfspace $u_{x}<0$. It satisfies

$$
u_{x}^{2}=p(u) \stackrel{\text { def }}{=} \frac{u^{2}\left(3 u^{2}-4(1+a) u+6 a\right)}{6}=\frac{u^{2}\left(u-u_{-}\right)\left(u-u_{+}\right)}{2},
$$

where

$$
a<u_{-}=\frac{2(1+a)}{3}\left(1-\sqrt{1-\frac{9 a}{2(1+a)^{2}}}\right)<1<u_{+}=\frac{2(1+a)}{3}\left(1+\sqrt{1-\frac{9 a}{2(1+a)^{2}}}\right) .
$$

Rather than analyze the boundary-value problem

$$
\begin{gathered}
d^{2} \phi / d x^{2}-\left(3 u_{\mathrm{eq}}^{2}(x)-2(1+a) u_{\mathrm{eq}}(x)+a\right) \phi(x)=\mu a \phi(x), \quad x>0, \\
\frac{d \phi}{d x}(0)=0 \text { and } \lim _{x \rightarrow \infty} \phi(x)=0
\end{gathered}
$$

directly, it is convenient to introduce the change of variable generated by the equilibrium problem, namely

$$
x=\int_{u}^{u_{-}} \frac{d \eta}{p^{1 / 2}(\eta)}, \quad 0<u<u_{-}
$$




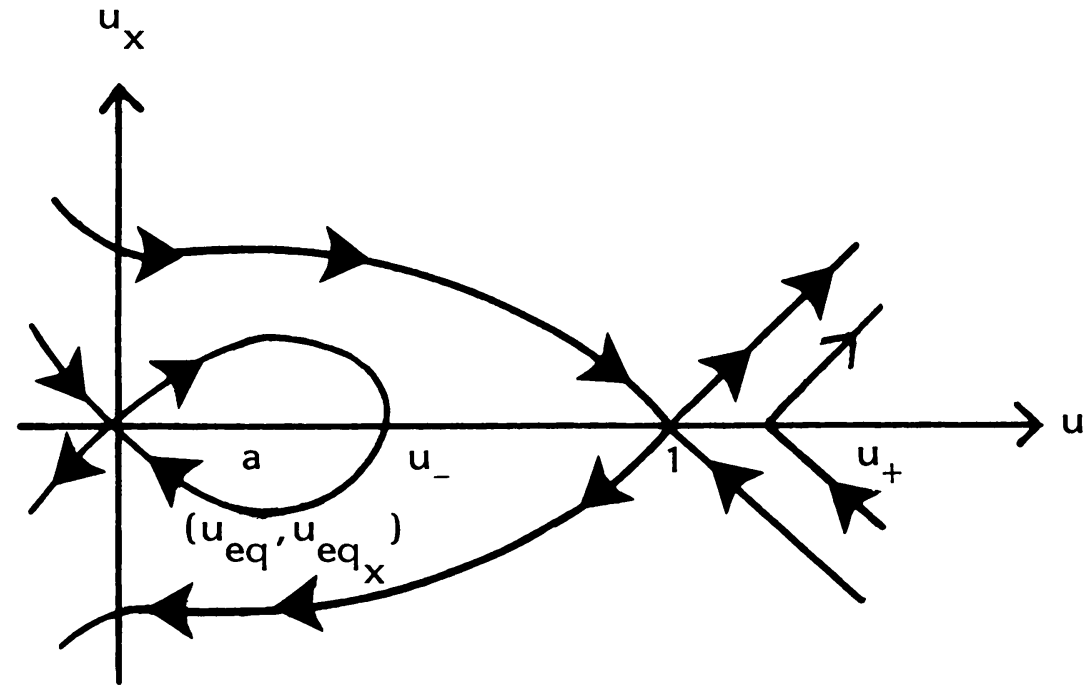

Fig. 1 .

If we then regard $\phi$ as a function of $u$, it is a simple matter to show that the problem (DE) $)_{\mathbf{V a r}}$ and $(\mathrm{BC})$ transforms into

$$
\begin{array}{cc}
p^{1 / 2}(u) \frac{d}{d u}\left(p^{1 / 2}(u) \frac{d \phi}{d u}\right)-\frac{p^{(2)}(u)}{2} \phi=\mu a \phi, \quad 0<u<u_{-}, & (\mathrm{DE})_{\mathrm{Var}} \\
\lim _{\varepsilon \rightarrow 0^{+}} p^{1 / 2}\left(u_{-}-\varepsilon\right) \frac{d \phi}{d u}\left(u_{-}-\varepsilon\right)=0 \text { and } \lim _{u \rightarrow 0^{+}} \phi(u)=0 . & \text { (BC) }
\end{array}
$$

Again,

$$
\begin{gathered}
p(u)=\frac{u^{2}\left(3 u^{2}-4(1+a) u+6 a\right)}{6}, \quad p^{(1)}(u)=2\left(u^{3}-(1+a) u^{2}+a u\right), \\
p^{(2)}(u)=2\left(3 u^{2}-2(1+a) u+a\right) .
\end{gathered}
$$

For each $\mu \geq 0$ we let $\phi_{1}(u, \mu)$ and $\phi_{2}(u, \mu)$ be the unique solutions of $(\mathrm{DE})_{\mathbf{V}_{a r}}$ satisfying the terminal conditions

$$
\begin{array}{ll}
\lim _{\varepsilon \rightarrow 0^{+}} \phi_{1}\left(u_{-}-\varepsilon, u\right)=0, & \lim _{\varepsilon \rightarrow 0^{+}} p^{1 / 2}\left(u_{-}-\varepsilon\right) \frac{d \phi_{1}}{d u}\left(u_{-}-\varepsilon, \mu\right)=1, \\
\lim _{\varepsilon \rightarrow 0^{+}} \phi_{2}\left(u_{-}-\varepsilon, \mu\right)=1, & \lim _{\varepsilon \rightarrow 0^{+}} p^{1 / 2}\left(u_{-}-\varepsilon\right) \frac{d \phi_{2}}{d u}\left(u_{-}-\varepsilon, \mu\right)=0 .
\end{array}
$$

When $\mu=0, \phi_{1}$ and $\phi_{2}$ may be calculated explicitly. The result is

$$
\phi_{1}(u, 0)=\frac{2 p^{1 / 2}(u)}{p^{(1)}\left(u_{-}\right)}, \quad \phi_{2}(u, 0)=1+\frac{p^{1 / 2}(u)}{2} \int_{u}^{u_{-}}\left(p^{(1)}\left(u_{-}\right)-p^{(1)}(s)\right) \frac{d s}{p^{3 / 2}(s)}
$$

for $0<u<u_{-}$. The function $\phi_{2}(u, 0)$ has one zero in $\left(0, u_{-}\right)$, call it $u_{2}(0)$, and at this point $\left(d \phi_{2} / d u\right)\left(u_{2}(0), 0\right)>0$. Moreover, $\lim _{u \rightarrow 0^{+}} \phi_{2}(u, 0)=-\infty$. For $0<\mu$ and $\mu$ small, the function $\phi_{2}(\cdot, \mu)$ is endowed with similar properties, namely a single zero $u_{2}(\mu)$ in $\left(0, u_{-}\right)$with 
the property that $\left(d \phi_{2} / d u\right)\left(u_{2}(\mu), u\right)>0$. The function $\mu \rightarrow u_{2}(\mu)$ satisfies $\left(d u_{2} / d \mu\right)(\mu)<0$ and $\phi_{2}(\cdot, \mu)$ obeys the limit relation $\lim _{u \rightarrow 0^{+}} \phi_{2}(u, \mu)=-\infty$. These properties persist so long as $u_{2}(\mu)>0$. This leads us to define $\bar{\mu}$ as the supremum of those numbers $\mu$ such that $u_{2}(\mu)>0$. Clearly, if $\bar{\mu}$ is finite, then $\bar{\mu} a$ is an eigenvalue of the variational operator with eigenfunction $\phi_{2}(\cdot, \bar{\mu})$. That $\bar{\mu}$ is finite follows from the observation that for $\mu>$ $-p^{(2)}\left(u_{-}\right) / 2,\left(d \phi_{2} / d u\right)(u, \mu) \leq 0$ and $\phi_{2}(u, \mu) \geq 1$ for $0<u \leq u_{-}$. That there are no eigenvalues $\mu a$ with $\mu>\bar{\mu}$ follows from the observation that the associated eigenfunction $\phi_{2}(\cdot$, $\mu$ ) would have to satisfy

$$
0=\int_{0}^{u-} \frac{p^{(2)}(u) \phi_{2}(u, \mu) \phi_{2}(u, \bar{\mu})}{p^{1 / 2}(u)} d u
$$

and thus have a zero in $\left(0, u_{-}\right)$, whereas the integral identity satisfied by $\phi_{2}(\cdot, \mu)$, namely

$$
\begin{aligned}
& \phi_{2}(u, \mu)=\phi_{2}(u, \bar{\mu})+(\mu-\bar{\mu}) \phi_{2}(u, \bar{\mu}) \\
& \times \int_{u}^{u-}\left[\frac{1}{p^{1 / 2}(t) \phi_{2}^{2}(t, \bar{\mu})} \int_{t}^{u_{-}} \frac{\phi_{2}(s, \mu) \phi_{2}(s, \bar{\mu})}{p^{1 / 2}(s)} d s\right] d t,
\end{aligned}
$$

and the fact that $\phi_{2}(u, \bar{\mu})>0$ for $0<u \leq u_{-}$lead to the contradictary conclusion that for $\mu>\bar{\mu}$ the function $\phi_{2}(\cdot, \mu)$ is positive on $\left(0, u_{-}\right]$.

3. Eigenvalue estimates. Our goal in this section is to find a solution of $(\mathrm{DE})_{\mathrm{V}_{\mathrm{ar}}}$ and (BC) of the form:

$$
\phi=\sum_{n=0}^{\infty}\left(\prod_{k=0}^{n} \rho_{k}\right) u^{n+\lambda} \quad \text { where } \rho_{0}=1 \text { and } \lambda>1 .
$$

Such a solution clearly meets the boundary condition at $u=0$. Moreover, if $\lambda>1$ may be chosen so that $\rho_{\infty}=\lim _{n \rightarrow \infty} \rho_{n}$ satisfies

$$
\left|\rho_{\infty} u_{-}\right|<1,
$$

then the series for $u^{-\lambda} \phi(u)$ will be convergent on the interval $\left(-1 /\left|\rho_{\infty}\right|, 1 /\left|\rho_{\infty}\right|\right)$ with $1 /\left|\rho_{\infty}\right|>u_{-}$, and thus the function $\phi(\cdot)$ will satisfy the boundary condition

$$
\lim _{\varepsilon \rightarrow 0^{+}} p^{1 / 2}\left(u_{-}-\varepsilon\right) \frac{d \phi}{d u}\left(u_{-}-\varepsilon\right)=0
$$

automatically.

Insertion of the representation (3.1) into the variational equation

$$
\begin{aligned}
& p^{1 / 2}(u) \frac{d}{d u}\left(p^{1 / 2}(u) \frac{d \phi}{d u}\right)-\left(\frac{p^{(2)}(u)}{2}+\mu a\right) \phi \\
&=\frac{\left.3 u^{4}-4(1+a) u^{3}+6 a u^{2}\right)}{6} \frac{d^{2} \phi}{d u^{2}}-\left(u^{3}-(1+a) u^{2}+a u\right) \frac{d \phi}{d u} \\
&-\left(3 u^{2}-2(1+a) u+(\mu+1) a\right) \phi=0
\end{aligned}
$$


yields the following relationships amongst the parameters $\lambda$ and $\mu$ and the coefficients $\rho_{n}$, $n=1,2, \ldots$ :

$$
\begin{gathered}
\mu=\lambda^{2}-1, \\
\rho_{0}=1, \\
\rho_{1}=\frac{2(1+a)(\lambda-3 / 2)(\lambda+2)}{3 a(1+2 \lambda)},
\end{gathered}
$$

and for $n \geq 2$

$$
a(n+2 \lambda) n \rho_{n} \rho_{n-1}-\frac{2(1+a)}{3}\left(n+\lambda-\frac{5}{2}\right)(n+\lambda+1) \rho_{n-1}+\frac{(n+\lambda-4)(n+\lambda+1)}{2}=0 .
$$

The possible limits of the sequence $\left\{\rho_{n}(\lambda)\right\}_{n=0}^{\infty}$ are the roots

$$
\underline{\rho}=\frac{(1+a)}{3 a}\left(1-\sqrt{1-\frac{9 a}{2(1+a)^{2}}}\right)<\bar{\rho}=\frac{(1+a)}{3 a}\left(1+\sqrt{1-\frac{9 a}{2(1+a)^{2}}}\right)
$$

of the polynomial equation

$$
a \rho^{2}-\frac{2(1+a)}{3} \rho+\frac{1}{2}=0 .
$$

These roots are related to the nonzero roots $u_{-}<u_{+}$of $p(u)$ (see (2.1) and (2.2)) by

$$
\underline{\rho}=\frac{1}{u_{+}} \text {and } \bar{\rho}=\frac{1}{u_{-}} \text {. }
$$

The results of section 2 imply there is at most one $\lambda>1$ such that $\lim _{n \rightarrow \infty} \rho_{n}(\lambda)=\rho$. Our task is to show how to find such a $\lambda$.

For $N \geq 3$ and $\lambda \in(1,2]$ we let

$$
\begin{aligned}
& R_{N}^{N}(\lambda)=\underline{\rho}, \quad \text { and } \\
& \qquad R_{n-1}^{N}(\lambda)=\frac{3(n+\lambda-4)}{4(1+a)(n+\lambda-5 / 2)} \frac{1}{\left(1-\frac{3 a}{2(1+a)} \frac{n(n+2 \lambda)}{(n+\lambda-5 / 2)(n+\lambda+1)} R_{n}^{N}(\lambda)\right)}, \\
& 2 \leq n \leq N .
\end{aligned}
$$

It is easily checked that the numbers $\left\{R_{n}^{N}(\lambda)\right\}_{n=1}^{N}$ satisfy $(3.4)_{n}$ for indices $n=2,3, \ldots, N$. To guarantee that (3.4) ${ }_{1}$ is satisfied, we must show that for each $N \geq 3$ there is a number $\lambda_{N}$ such that

$$
R_{1}^{N}\left(\lambda_{N}\right)=\frac{2(1+a)\left(\lambda_{N}-3 / 2\right)\left(\lambda_{N}+2\right)}{3 a\left(1+2 \lambda_{N}\right)}
$$

We shall discuss the solvability of (3.8) $)_{N}$ later, but first we record some properties of the sequence $\left\{R_{n}^{N}(\lambda)\right\}_{n=1}^{N}$. 
LEMmA 3.1. For each $N \geq 3$ and $\lambda \in(1,2]$, the sequence $\left\{R_{n}^{N}(\lambda)\right\}_{n=1}^{N}$ satisfies

$$
\begin{gathered}
0<R_{2}^{N}(\lambda)<R_{3}^{N}(\lambda)<\cdots<R_{n-1}^{N}(\lambda)<R_{N}^{N}(\lambda)=\underline{\rho}, \\
R_{n}^{N+1}(\lambda)<R_{n}^{N}(\lambda), \quad 2 \leq n \leq N .
\end{gathered}
$$

Proof. We start by examining the properties of the function

$$
F(\mu, \lambda, \rho) \stackrel{\text { def }}{=} \frac{3(\mu-4)}{4(1+a)(\mu-5 / 2)} \frac{1}{\left(1-\frac{3 a}{2(1+a)} \frac{\mu^{2}-\lambda^{2}}{\left(\mu^{2}-3 \mu / 2-5 / 2\right)} \rho\right)}
$$

when

$$
4 \leq \mu, \quad 1 \leq \lambda \leq 2, \quad 0 \leq \rho \leq \underline{\rho}=\left(\frac{1+a}{3 a}\right)\left(1-\sqrt{1-\frac{9 a}{2(1+a)^{2}}}\right), \quad 0<a<\frac{1}{2} .
$$

The sequence $\left\{R_{n}^{N}(\lambda)\right\}_{n=1}^{N}$ is generated by the recurrence relation

$$
R_{n-1}^{N}(\lambda)=F\left(n+\lambda, \lambda, R_{n}^{N}(\lambda)\right), \quad 2 \leq n \leq N \quad \text { and } \quad R_{N}^{N}(\lambda)=\rho .
$$

The following facts are easily verified for parameters $\mu, \lambda, \rho$, and $a$ satisfying (3.12):

$$
\begin{aligned}
0<\frac{\mu^{2}-\lambda^{2}}{2\left(\mu^{2}-\frac{3 \mu}{2}-\frac{5}{2}\right)} \leq 1, \quad 0<\frac{9 a}{2(1+a)^{2}}<1, \quad 0<\frac{a \rho}{(1+a)}<\frac{1}{3}, \\
1-\frac{3 a}{2(1+a)} \frac{\left(\mu^{2}-\lambda^{2}\right)}{\left(\mu^{2}-\frac{3 \mu}{2}-\frac{5}{2}\right)} \rho \geq 1-\frac{3 a}{2(1+a)} \frac{\left(\mu^{2}-\lambda^{2}\right)}{\left(\mu^{2}-\frac{3 \mu}{2}-\frac{5}{2}\right)} \rho>0 .
\end{aligned}
$$

The constraints (3.12) and (3.14) 4 guarantee that

$$
0<F(\mu, \lambda, \rho) \leq F(\mu, \lambda, \underline{\rho}) \text { and } 0<\frac{\partial F}{\partial \rho}(\mu, \lambda, \rho)
$$

for $4<\mu, 1<\lambda \leq 2,0 \leq \rho \leq \underline{\rho}$, and $0<a<\frac{1}{2}$. When $\mu=4, F(4, \lambda, \rho)=\partial F / \partial \rho(4, \lambda, \rho)=0$. We also have

$$
F(\mu, \lambda, \underline{\rho})<\underline{\rho}=\frac{3}{4(1+a)} \frac{1}{\left(1-\frac{3 a \underline{\rho}}{2(1+a)}\right)} .
$$

To establish the veracity of (3.16) it suffices to show that

$$
(\mu-4)\left(1-\frac{3 a \rho}{2(1+a)}\right)<\left(\mu-\frac{5}{2}\right)\left(1-\frac{3 a \rho}{2} \frac{\left(\mu^{2}-\lambda^{2}\right)}{\left(\mu^{2}-\frac{3 \mu}{2}-\frac{5}{2}\right)}\right)
$$

and this in turn is true if and only if

$$
\frac{a \varrho}{1+a}<\frac{\mu+1}{4+3 \mu-\lambda^{2}}=\frac{\mu+1}{3(\mu+1)+\left(1-\lambda^{2}\right)} .
$$


But (3.12) and (3.14) imply that

$$
\frac{a \varrho}{1+a}<\frac{1}{3} \text { and } \frac{1}{3} \leq \frac{\mu+1}{4+3 \mu-\lambda^{2}}
$$

and thus (3.16) is true.

The next inequality establishes the monotonicity of $F$ in $\mu$ on $4 \leq \mu, 1 \leq \lambda \leq 2$, $0 \leq \rho \leq \rho$, and $0<a<\frac{1}{2}$ :

$$
0<\frac{\partial F}{\partial \mu}(\mu, \lambda, \rho)
$$

To establish (3.19) it suffices to show that

$$
\frac{3}{2\left(\mu-\frac{5}{2}\right)^{2}}\left(1-\frac{3 a \rho\left(\mu^{2}-\lambda^{2}\right)}{2(1+a)\left(\mu-\frac{5}{2}\right)(\mu+1)}\right)+\frac{3 a \rho}{4(1+a)}\left(\frac{\mu-4}{\mu-\frac{5}{2}}\right) \frac{\partial}{\partial \mu}\left(\frac{3 \mu+5-2 \lambda^{2}}{\left(\mu-\frac{5}{2}\right)(\mu+1)}\right)>0 .
$$

Moreover, (3.20) is true if and only if

$$
\begin{aligned}
\frac{3}{2}-\frac{3 a \rho}{4(1+a)}\left[\frac{3\left(\mu^{2}-\lambda^{2}\right)}{\left(\mu-\frac{5}{2}\right)(\mu+1)}+\frac{\left(3 \mu+5-2 \lambda^{2}\right)(\mu-4)}{\left(\mu-\frac{5}{2}\right)(\mu+1)}\right. & \left.+\frac{\left(3 \mu+5-2 \lambda^{2}\right)(\mu-4)}{(\mu+1)^{2}}-\frac{3(\mu-4)}{(\mu+1)}\right]>0
\end{aligned}
$$

and (3.21) is true if and only if

$$
\begin{aligned}
& \frac{a \rho}{1+a}<\frac{2 \mu^{3}-\mu^{2}-8 \mu-5}{6 \mu^{3}-3 \mu^{2}-\left(14+10 \lambda^{2}\right) \mu-40+25 \lambda^{2}} \\
& \quad=\frac{1}{3}+\frac{5\left(\lambda^{2}-1\right)(2 \mu-5)}{6 \mu^{3}-3 \mu^{2}-\left(14+10 \lambda^{2}\right) \mu-40+25 \lambda^{2}} .
\end{aligned}
$$

But

$$
\begin{gathered}
\frac{a \rho}{1+a} \leq \frac{a \rho}{1+a}<\frac{1}{3}, \quad 0 \leq \lambda^{2}-1, \quad 3 \leq 2 \mu-5, \\
6 \mu^{3}-3 \mu^{2}-\left(14-10 \lambda^{2}\right) \mu-40+25 \lambda^{2} \\
=3(\mu-\lambda)(\mu+\lambda)(\mu+1)+(\mu-4)\left(3 \mu^{2}+\left(10-4 \lambda^{2}\right) \mu+3 \lambda^{2}\right), \\
3 \mu^{2}+\left(10-4 \lambda^{2}\right) \mu+3 \lambda^{2} \geq \frac{25}{12}\left(\lambda^{2}-1\right)(5-2 \lambda)(5+2 \lambda) \geq 0, \\
\frac{1}{3}+\frac{5\left(\lambda^{2}-1\right)(2 \mu-5)}{6 \mu^{3}-3 \mu^{2}-\left(14+10 \lambda^{2}\right) \mu-40+25 \lambda^{2}} \geq \frac{1}{3}
\end{gathered}
$$

and therefore (3.22) holds for all $\mu, \lambda, \rho$, and $a$ satisfying (3.12).

We now turn our attention to the inequality (3.9). Eq. (3.13) with $n=N-1$ yields

$$
R_{N-1}^{N}(\lambda)=F(N+\lambda, \lambda, \underline{\rho})
$$

and (3.15) and (3.16) imply that $0<R_{N-1}^{N}(\lambda)<\underline{\rho}$. We now make the induction hypothesis that

$$
R_{k-1}^{N}(\lambda)<R_{k}^{N}(\lambda), \quad k=n+1, \ldots, N \text { and } n \geq 3
$$


The inequalities (3.15), (3.19), and (3.25) imply that

$$
0<F\left(n+\lambda, \lambda, R_{n}^{N}(\lambda)\right)<F\left(n+1+\lambda, \lambda, R_{n+1}^{N}(\lambda)\right)
$$

and (3.26) together with (3.13) yields

$$
R_{n-1}^{N}(\lambda)<R_{n}^{N}(\lambda)
$$

which is the induction hypothesis at stage $n$.

The inequality (3.10) follows from a similar argument and the observation that

$$
R_{N}^{N+1}(\lambda)<\underline{\rho}=R_{N}^{N}(\lambda)
$$

LEMmA 3.2. For each integer $N \geq 3$, the equation $(3.8)_{N}$ has a solution in $\lambda_{N} \in\left(1, \frac{3}{2}\right)$. Moreover, the numbers $\lambda_{N}$ satisfy $\lambda_{N}<\lambda_{N+1}$.

Proof. We start with the observation that solving $(3.8)_{N}$ is equivalent to solving

$$
R_{2}^{N}(\lambda)=\rho_{2}(\lambda) \stackrel{\text { def }}{=} \frac{(1+a)}{6 a} \frac{(\lambda+3)\left(\lambda-\frac{1}{2}\right)}{(\lambda+1)}-\frac{3(\lambda+3)(2 \lambda+1)(\lambda-2)}{16(1+a)(\lambda+2)(\lambda+1)\left(\lambda-\frac{3}{2}\right)} .
$$

We treat the case where $N=3$ first. The function

$$
R_{2}^{3}(\lambda) \stackrel{\text { def }}{=} \frac{3(\lambda-1)}{4(1+a)\left(\lambda-\frac{1}{2}\right)} \frac{1}{\left(1-\frac{a \rho}{(1+a)} \frac{9(3+2 \lambda)}{2\left(\lambda-\frac{1}{2}\right)(\lambda+4)}\right)}
$$

satisfies $\lim _{\lambda \rightarrow 1} R_{2}^{3}(\lambda)=0$ and $R_{2}^{3}(\lambda)>0$ for $\lambda>1$, whereas $\rho_{2}(\cdot)$ satisfies $\lim _{\lambda \rightarrow 1} \rho_{2}(\lambda)=$ $(2-a)(1-2 a) / 6 a(1+a)>0,0<a<\frac{1}{2}$, and $\lim _{\lambda \rightarrow(3 / 2)^{-}} \rho_{2}(\lambda)=-\infty$. Thus there is a number $\lambda_{3} \in\left(1, \frac{3}{2}\right)$ such that $(3.29)_{3}$ holds. We now suppose that $(3.29)_{k}$ is solvable for $k=3,4, \ldots, N$ and that the solutions satisfy $\lambda_{k} \in\left(1, \frac{3}{2}\right)$ and $\lambda_{k}<\lambda_{k+1}, k=3,4, \ldots, N-1$. The inequality (3.10) $)_{2}$ and Eq. (3.29) $)_{N}$ imply that $\rho_{2}\left(\lambda_{N}\right)=R_{2}^{N}\left(\lambda_{N}\right)>R_{2}^{N+1}\left(\lambda_{N}\right)>0$ and this, combined with $\lim _{\lambda \rightarrow(3 / 2)^{-}} \rho_{2}(\lambda)=-\infty$, implies that $(3.29)_{N+1}$ has a solution $\lambda_{N+1} \in\left(\lambda_{N}, \frac{3}{2}\right)$. This is the induction hypothesis at stage $N$ and completes the proof.

If we now let $\left\{\rho_{n}(\lambda)\right\}_{n=0}^{\infty}$ be the coefficient sequence defined by (3.4), then for each integer $N \geq 3$

$$
R_{n}^{N}\left(\lambda_{N}\right)=\rho_{n}\left(\lambda_{N}\right), \quad 1 \leq n \leq N
$$

If we let $\lambda_{\infty} \stackrel{\text { def }}{=} \lim _{N \rightarrow \infty} \lambda_{N}$, then (3.30) and the results of Lemma 3.1 imply that for $n \geq 2$

$$
0<\rho_{n}\left(\lambda_{\infty}\right)<\rho_{n+1}\left(\lambda_{\infty}\right)<\underline{\rho}=\frac{1}{u_{+}}
$$

and thus the function

$$
\phi(u)=\sum_{n=0}^{\infty}\left(\prod_{k=0}^{n} \rho_{k}\left(\lambda_{\infty}\right)\right) u^{n+\lambda_{\infty}}
$$

is the eigenfunction of the variational operator which satisfies the boundary conditions (BC) at $u=0$ and $u=u_{-}$. Again, the function $u^{-\lambda_{\infty}} \phi(u)$ is analytic on $\left(-u_{+}, u_{+}\right)$. 


\section{REFERENCES}

[1] Paul C. Fife, Mathematical aspects of reacting and diffusing systems, Lecture Notes in Biomathematics 28, Springer, Berlin, 1979

[2] Paul C. Fife and J. B. Mcleod, The approach of nonlinear diffusion equations to traveling front solutions, Arch. Rat. Mech. Anal. 65, 335-361 (1977)

[3] D. G. Aronson and H. F. Weinberger, Nonlinear diffusion in population genetics, combustion, and nerve propagation, in Proceedings of the Tulane Program in Partial Differential Equations and Related Topics, Lecture Notes in Mathematics 446, 5-49, Springer, Berlin, 1975

[4] M. D. Bramson, Maximal displacement of branching Brownian motion, Ph.D. Thesis, Cornell University, 1977 